\title{
ON THE MOORE-PENROSE INVERSE IN RINGS WITH INVOLUTION
}

\author{
DIJANA MOSIĆ
}

Received 12 December, 2015

\begin{abstract}
We study equivalent conditions to those given in Penrose equations for an element to be the Moore-Penrose inverse of a given element in a ring with involution, using the concept of EP, normal, bi-EP, bi-normal, $l$-(or $r$-)quasi-EP, $l$-(or $r$-)quasi-normal and *-cancellable elements. The mentioned conditions are weaker that the one of being self-adjoint.
\end{abstract}

2010 Mathematics Subject Classification: 16W10; 15A09; 46L05

Keywords: Moore-Penrose inverse, EP elements, normal elements, ring with involution

\section{INTRODUCTION}

Let $\mathcal{R}$ be an associative ring with the unit 1 . An involution $a \mapsto a^{*}$ in a ring $\mathcal{R}$ is an anti-isomorphism of degree 2 , that is,

$$
\left(a^{*}\right)^{*}=a, \quad(a+b)^{*}=a^{*}+b^{*}, \quad(a b)^{*}=b^{*} a^{*} .
$$

An element $a \in \mathcal{R}$ is self-adjoint if $a^{*}=a$. An element $a \in \mathcal{R}$ satisfying $a a^{*}=a^{*} a$ is called normal.

The Moore-Penrose inverse of $a \in \mathcal{R}$ is the element $x \in \mathcal{R}$, if the following Penrose equations hold [7]:
(1) $a \times a=a$,
(2) $x a x=x$,
(3) $(a x)^{*}=a x$,
(4) $(x a)^{*}=x a$.

There is at most one $x$ such that above conditions hold, and such $x$ is denoted by $a^{\dagger}$. The set of all Moore-Penrose invertible elements of $\mathcal{R}$ will be denoted by $\mathcal{R}^{\dagger}$. In the last decades, the Moore-Penrose inverse has found a wide range of applications in many areas of science and became a useful tool for dealing with optimization problems, data analysis, the solution of linear integral equations, etc.

If $\delta \subseteq\{1,2,3,4\}$ and $x$ satisfies the equations $(i)$ for all $i \in \delta$, then $x$ is a $\delta$-inverse of $a$. The set of all $\delta$-inverse of $a$ is denote by $a\{\delta\}$. Notice that $a\{1,2,3,4\}=\left\{a^{\dagger}\right\}$. Recall that $x \in a\{1,4\}$ if and only if $x^{*} \in a^{*}\{1,3\}$.

The following result is frequently used in the rest of the paper.

Theorem 1 ([1]). For any $a \in \mathcal{R}^{\dagger}$, the following is satisfied: 
(a) $\left(a^{\dagger}\right)^{\dagger}=a$;

(b) $\left(a^{*}\right)^{\dagger}=\left(a^{\dagger}\right)^{*}$.

An element $a \in \mathcal{R}$ is said to be EP if $a \in \mathcal{R}^{\dagger}$ and $a a^{\dagger}=a^{\dagger} a$. Observe that $a$ is EP if and only if $a^{*}$ is EP.

We recall the reader that $a \in \mathcal{R}$ is called (i) bi-normal if $a a^{*} a^{*} a=a^{*} a a a^{*}$; (ii) bi-EP if $a \in \mathcal{R}^{\dagger}$ and $a a^{\dagger} a^{\dagger} a=a^{\dagger} a a a^{\dagger}$; (iii) $l$-quasi-normal if $a a^{*} a=a^{*} a a$; (iv) $r$-quasi-normal if $a a a^{*}=a a^{*} a$; (v) $l$-quasi-EP if $a \in \mathcal{R}^{\dagger}$ and $a a^{\dagger} a=a^{\dagger} a a$; (vi) $r$-quasi-EP if $a \in \mathcal{R}^{\dagger}$ and $a a a^{\dagger}=a a^{\dagger} a[2,4,6]$.

An element $a \in \mathcal{R}$ is: left *-cancellable if $a^{*} a x=a^{*} a y$ implies $a x=a y$; it is right *-cancellable if $x a a^{*}=y a a^{*}$ implies $x a=y a$; and it is *-cancellable if it is both left and right $*$-cancellable. Notice that $a$ is left *-cancellable if and only if $a^{*}$

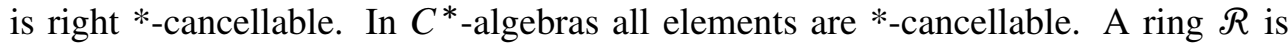
called *-reducing if every element of $\mathcal{R}$ is $*$-cancellable. This is equivalent to the implication $a^{*} a=0 \Rightarrow a=0$ for all $a \in \mathcal{R}$.

Malik and Thome [6] investigated weaker conditions than those given by Penrose for an operator to be the Moore-Penrose inverse of a given bounded linear operator between two Hilbert spaces, using operator matrices.

In this paper, applying a purely algebraic technique, we characterize the MoorePenrose inverse of an element in a ring with involution in terms of EP, normal, bi-EP, bi-normal, $l$-quasi-EP, $r$-quasi-EP, $l$-quasi-normal, $r$-quasi-normal and $*_{\text {- }}$ cancellable elements, generalizing the results from [6]. Thus, we show that the properties of operator matrices are not necessary for the characterization of the MoorePenrose inverse.

\section{Results}

In the first theorem, if $x$ is an 1-inverse of $a$, we present necessary and sufficient conditions for $x$ to be a 3-inverse of $a$.

Theorem 2. Let $a \in \mathcal{R}$ such that $a\{1\} \neq \varnothing$. If $x \in a\{1\}$, then the following statements are equivalent:

(i) ax is self-adjoint;

(ii) $a x$ is EP;

(iii) ax is right *-cancellable and normal;

(iv) ax is bi-EP;

(v) ax is *-cancellable and bi-normal;

(vi) ax is l-quasi-EP;

(vii) ax is r-quasi-EP;

(viii) ax is left *-cancellable and l-quasi-normal;

(ix) ax is right *-cancellable and r-quasi-normal.

Proof. (i) $\Rightarrow$ (ii)-(ix): Because $a x$ is self-adjoint idempotent, we deduce that $a x \in \mathcal{R}^{\dagger}$ and $(a x)^{\dagger}=a x$. So, the statements (ii)-(ix) hold. 
(ii) $\Rightarrow$ (i): Since $a x$ is EP, then $a x \in \mathcal{R}^{\dagger}$ and $(a x)^{\dagger} a x=a x(a x)^{\dagger}$. Thus,

$$
a x=\operatorname{ax}(a x)^{\dagger} a x=\operatorname{axax}(a x)^{\dagger}=\operatorname{ax}(a x)^{\dagger}
$$

is self-adjoint.

(iii) $\Rightarrow$ (i): If $a x$ is right *-cancellable and normal, then $(a x) *$ is left *-cancellable and $a x(a x)^{*}=(a x)^{*} a x$. Multiplying this equality by $a x$ from the left side, it follows

$$
a x(a x)^{*}=a x(a x)^{*} a x .
$$

Using *-cancellation, we get $(a x)^{*}=(a x)^{*} a x$, i.e. $a x=(a x)^{*} a x$ is self-adjoint.

(iv) $\Rightarrow$ (i): Suppose that $a x$ is bi-EP, that is, $a x \in \mathcal{R}^{\dagger}$ and

$$
\operatorname{ax}(a x)^{\dagger}(a x)^{\dagger} a x=(\operatorname{ax})^{\dagger} \operatorname{axax}(a x)^{\dagger} .
$$

Now, we get

$$
\begin{aligned}
{\left[(a x)^{\dagger}\right]^{*} } & =\left[(a x)^{\dagger} a x(a x)^{\dagger}\right]^{*}=\left[(a x)^{\dagger} \operatorname{axax}(a x)^{\dagger}\right]^{*} \\
& =\left[a x(a x)^{\dagger}(a x)^{\dagger} a x\right]^{*}=(a x)^{\dagger} \operatorname{axax}(a x)^{\dagger} \\
& =(a x)^{\dagger} a x(a x)^{\dagger}=(a x)^{\dagger}
\end{aligned}
$$

which implies $(a x)^{*}=\left(\left[(a x)^{\dagger}\right]^{*}\right)^{\dagger}=\left[(a x)^{\dagger}\right]^{\dagger}=a x$.

(v) $\Rightarrow$ (i): Let $a x$ be *-cancellable and bi-normal. Then

$$
a x(a x)^{*}(a x)^{*} a x=(a x)^{*} \operatorname{axax}(a x)^{*}
$$

gives

$$
a x(a x)^{*} a x=(a x)^{*} a x(a x)^{*} .
$$

Multiplying the previous equality by $a x$ from the left side, we obtain

$$
a x(a x)^{*} a x=a x(a x)^{*} a x(a x)^{*} .
$$

Since $a x$ and $(a x)^{*}$ are *-cancellable, by (2.1), $a x=a x(a x)^{*}$ is self-adjoint.

(vi) $\Rightarrow$ (i): Because $a x$ is $l$-quasi-EP, we have that $a x \in \mathcal{R}^{\dagger}$ and

$$
\operatorname{ax}(a x)^{\dagger} a x=(a x)^{\dagger} \operatorname{axax}
$$

implying $a x=(a x)^{\dagger} a x$ is self-adjoint.

(vii) $\Rightarrow$ (i): Similarly as (vi) $\Rightarrow$ (i).

(viii) $\Rightarrow$ (i): Assume that $a x$ is left $*$-cancellable and $l$-quasi-normal. We deduce that $(a x)^{*}$ is right $*$-cancellable and

$$
a x(a x)^{*} a x=(a x)^{*} a x a x=(a x)^{*} a x,
$$

which yield $a x(a x)^{*}=(a x)^{*}$. Hence, $a x(a x)^{*}=a x$ is self-adjoint.

(ix) $\Rightarrow$ (i): In the same way as in the part (viii) $\Rightarrow$ (i).

The following result can be checked similarly as Theorem 2 .

Corollary 1. Let $a \in \mathcal{R}$ such that $a\{1\} \neq \varnothing$. If $x \in a\{1\}$, then the following statements are equivalent: 
(i) ax is self-adjoint;

(ii) $a x$ is $E P$;

(iii) ax $\in \mathcal{R}^{\dagger}$ and ax is normal;

(iv) $a x$ is bi-EP;

(v) ax $\in \mathcal{R}^{\dagger}$ and ax is bi-normal;

(vi) ax is $l$-quasi-EP;

(vii) ax is r-quasi-EP;

(viii) ax $\in \mathcal{R}^{\dagger}$ and ax is l-quasi-normal;

(ix) $a x \in \mathcal{R}^{\dagger}$ and ax is r-quasi-normal.

Proof. Since $a x \in \mathcal{R}^{\dagger}$, by [5, Theorem 5.3], we deduce that $a x$ is *-cancellable. The rest of this proof follows as in the proof of Theorem 2.

For an 1-inverse $x$ of $a$, several equivalent conditions which insure that $x$ is 4inverse of $a$ are given now.

Theorem 3. Let $a \in \mathcal{R}$ such that $a\{1\} \neq \varnothing$. If $x \in a\{1\}$, then the following statements are equivalent:

(i) $x a$ is self-adjoint;

(ii) $x a$ is $E P$;

(iii) $x a$ is left*-cancellable and normal;

(iv) $x a$ is $b i-E P$;

(v) $x a$ is *-cancellable and bi-normal;

(vi) $x a$ is $r$-quasi-EP;

(vii) $x a$ is $l$-quasi-EP;

(viii) $x$ a is right *-cancellable and $r$-quasi-normal;

(ix) $x a$ is left *-cancellable and l-quasi-normal.

Proof. For $x \in a\{1\}$ such that $x a$ satisfies any of the conditions (i)-(ix), we have that $x^{*} \in a^{*}\{1\}$ such that $a^{*} x^{*}$ satisfies any of the conditions (i)-(ix) of Theorem 2 . Applying Theorem 2, we verify this result.

In the main result of this article, weaker conditions than those given in Penrose equations for an element $x$ to be the Moore-Penorose inverse of $a$ are given.

Theorem 4. Let $a \in \mathcal{R}$ such that $a\{1,2\} \neq \varnothing$ and $x \in a\{1,2\}$. If ax satisfies any of the conditions (ii)-(ix) of Theorem 2 and xa satisfies any of the conditions (ii)-(ix) of Theorem 3, then $x$ is the Moore-Penrose inverse of a.

Proof. This result is a consequence of Theorem 2 and Theorem 3.

The preceding theorems hold in rings with involution assuming in some conditions that $a x$ and $x a$ are left or (and) right *-cancellable which is automatically satisfied in $C^{*}$-algebras and ${ }^{*}$-reducing rings. Thus, we recover the results in [6] for Hilbert space operators. 
In a unital $C^{*}$-algebra $\mathcal{A}$, since $a \in \mathcal{A}$ is Moore-Penrose invertible if and only if $a\{1\} \neq \varnothing$ (see [3]), we get the following result as a consequence of Theorem 4 .

Corollary 2. Let $a \in \mathcal{A}$ such that $a\{1\} \neq \varnothing$ and $x \in a\{1,2\}$. If both ax and $x a$ satisfy any of the statements:

(i) $E P$;

(ii) normal;

(iv) $b i-E P$;

(v) bi-normal;

(vi) $l-q u a s i-E P$;

(vii) $r$-quasi-EP;

(viii) l-quasi-normal;

(ix) $r$-quasi-normal,

then $x$ is the Moore-Penrose inverse of $a$.

By corresponding examples in [6], it is showed that conditions (i)-(ix) of Corollary 2 are weaker that one of being self-adjoint and can be adopted to define the MoorePenrose inverse.

\section{AKNOWLEDGEMENT}

The author is supported by the Ministry of Science, Republic of Serbia, grant No. 174007.

\section{REFERENCES}

[1] D. M. c and D. S. Djordjević, "Moore-penrose-invertible normal and hermitian elements in rings," Linear Algebra Appl., vol. 431, no. 5-7, pp. 732-745, 2009, doi: 10.1016/j.laa.2009.03.023.

[2] T. Furuta, Invitation to linear operators. New York: Taylor and Francis, 2001.

[3] R. E. Harte and M. Mbekhta, "On generalized inverses in $c^{*}$-algebras," Studia Math, vol. 103, pp. 71-77, 1992.

[4] R. E. Hartwig and K. Spindelböck, "Matrices for which $a^{*}$ and $a^{\dagger}$ commute," Linear Multilinear Algebra, vol. 14, pp. 241-256, 1984, doi: 10.1080/03081088308817561.

[5] J. J. Koliha and P. Patrí, "Elements of rings with equal spectral idempotents," J. Australian Math. Soc., vol. 72, pp. 137-152, 2002.

[6] S. B. Malik and N. Thome, "On a revisited moore-penrose inverse of a linear operator on hilbert spaces," 2017," doi: 10.2298/FIL1707927M.

[7] R. Penrose, "A generalized inverse for matrices," Proc. Cambridge Philos. Soc., vol. 51, pp. 406413, 1955, doi: 10.1017/S0305004100030401.

Author's address

\section{Dijana Mosić}

Faculty of Sciences and Mathematics, University of Niš, P.O. Box 224, 18000 Niš, Serbia

E-mail address: dijanaepmf.ni.ac.rs 